\title{
Right Sided Heart Failure-An Unusual Presentation of Aortic Valve Dehiscence
}

\section{Mohan Mallikarjuna Rao Edupuganti*}

EDivision of Cardiovascular Medicine, Department of Internal Medicine, University of Arkansas for Medical Sciences, 4301 West Markham Street, Little Rock,Arkansas, 72205 , USA

\begin{abstract}
Aortic dehiscence is an uncommon but serious complication of aortic valve surgery. The most common presentation is that of congestive heart failure and or cardiogenic shock. A number of other local complications can ensue due to involvement of the fibrous skeleton of the heart. We present a case where the dehiscence of the aortic valve resulted in elevated pulmonary arterial pressures and right sided heart failure due to the involvement of the right ventricular outflow tract. We review the literature focusing on the causes, summarize the diagnostic findings and the local complications that can result from aortic dehiscence. This complication of aortic valve dehiscence has not been reported so far.
\end{abstract}

Keywords: Aortic valve dehiscence; Echolucent space; Heart failure; Transesophageal echocardiogram

\section{Introduction}

Aortic dehiscence carries a high morbidity and mortality and requires an expeditious surgical replacement of the aortic valve in a hemodynamically compromised patient. Knowledge of the etiology, clinical and imaging features and potential local complications due to breakdown of the fibrous skeleton are important for timely management. Finally an understanding of the factors that need to be taken into consideration for planning a surgical approach and determining the optimal timing of intervention is necessary to ensure best possible outcomes.

\section{Case Presentation}

A 21 year old Caucasian male with a past medical history of bicuspid aortic valve, aortic stenosis and insufficiency and ascending aortic aneurysm underwent a Ross procedure with $26 \mathrm{~mm}$ pulmonary homograft valve placement and a $28 \mathrm{~mm}$ Hemashield graft for aortic aneurysm repair in June, 2013.

In February 2015, he presented with features of right sided heart failure of 4 weeks duration complaining of a progressively worsening shortness of breath on exertion pedal edema and abdominal swelling. He was referred to the Arkansas Children's hospital as an outpatient for further management.

On arrival he was hemodynamically stable with normal vital signs and temperature. General examination revealed bilateral pitting pedal edema, there were no stigmata of infective endocarditis, cardiac examination revealed a loud holosystolic murmur over the left sternal border and an elevated Jugular venous pressure measuring $12 \mathrm{~cm}$ above the sternal angle. Abdomen was distended and firm without tenderness. The remaining systems were normal on examination.

Echocardiographic examination revealed a borderline dilated left ventricle, the end diastolic diameter was $6.38 \mathrm{~cm}$, with a left ventricular ejection fraction $50 \%$, the right ventricle was moderately dilated with a reduced systolic function and markedly elevated right ventricular systolic pressure- $69.2 \mathrm{~mm} \mathrm{Hg}$, the right ventricular diameter at the base was $4.2 \mathrm{~cm}$. There was severe tricuspid valve regurgitation with severely dilated right atrium and loss of cooptation of the tricuspid valve leaflets. There was mild aortic regurgitation and no stenosis. The most striking feature was a giant pseudoaneurysm originating from the left ventricular outflow tract extending circumferentially around the aortic root and compressing the main pulmonary trunk. The aortic valve was elevated above the level of the annulus. There was a communication between the left and the right ventricular outflow tracts with the aortic flow entering the right ventricular outflow during ventricular systole. The dehiscence of the aortic valve and its displacement above the plane of the aortic annulus are best demonstrated on the parasternal long axis view on a transthoracic echocardiogram (Figure 1). Figure 1 shows the point of dehiscence and a large echolucent space (pseuodoaneurysm) around the dehiscence.

A Transesophageal echocardiogram was performed to confirm the findings and the pseuoduaneurysm is best demonstrated on the short axis view of the aortic valve (Figure 2). The large echolucent space surrounding the aortic valve in this view is the giant pseuodoaneurysm and it can be seen compressing the pulmonary valve and artery. Addition of color doppler to the short axis view demonstrates an abnormal communication between the outflow tracts of the left and right ventricles and blood flow into the pseudoaneurysm (Figure 3). A CT scan was performed to plan a surgical approach, a $3 \mathrm{D}$ reconstruction shows the extent of the pseudoaneurysm on the external surface of the heart (Figure 4).

The next day the patient underwent a Bentall procedure using a 31 $\mathrm{mm}$ valved conduit, closure of the fistula and pulmonary artery patch plasty with bovine pericardium. There were no intraoperative signs of infection. The procedure was successful and the patient tolerated the procedure well.

Histopathological exam revealed a dense fibrous tissue with hemorrhage, focal acute inflammation, and blood clot. There was myxoid degeneration of the aortic valve.

*Corresponding author: Mohan Mallikarjuna Rao Edupuganti, Division of Cardiovascular Medicine, Department of Internal Medicine, University of Arkansas for Medical Sciences, 4301 West Markham Street, Little Rock, Arkansas,72205, USA, Tel: 412-418-1111; E-mail: mmedupuganti@gmail.com

Received June 03, 2015; Accepted July 20, 2015; Published July 27, 2015

Citation: Edupuganti MMR (2015) Right Sided Heart Failure-An Unusual Presentation of Aortic Valve Dehiscence. J Health Med Informat 6: 195. doi:10.4172/21577420.1000195

Copyright: (c) 2015 Mallikarjuna M, et al. This is an open-access article distributed under the terms of the Creative Commons Attribution License, which permits unrestricted use, distribution, and reproduction in any medium, provided the original author and source are credited. 
Citation: Edupuganti MMR (2015) Right Sided Heart Failure-An Unusual Presentation of Aortic Valve Dehiscence. J Health Med Informat 6: 195. doi:10.4172/2157-7420.1000195

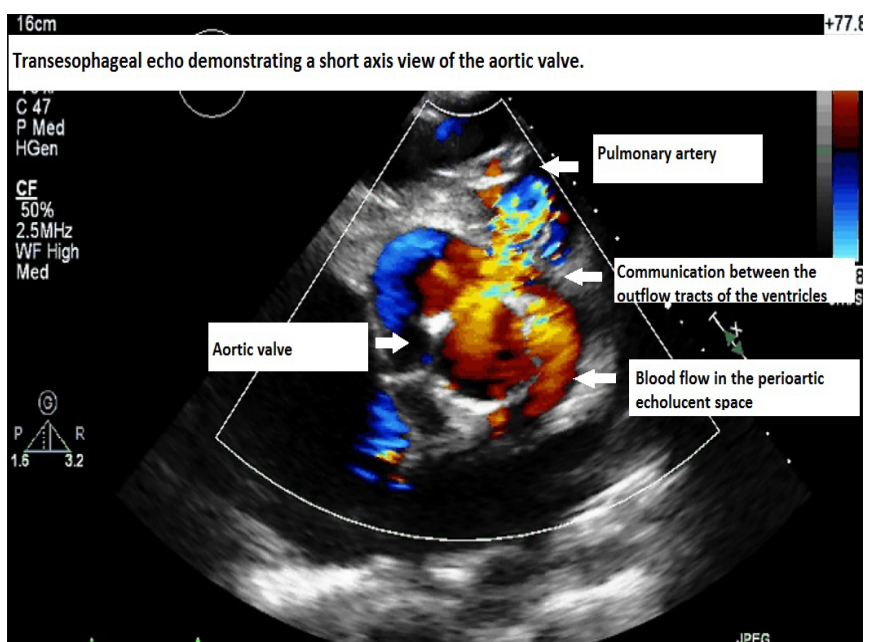

Figure 1: Parasternal long axis view of transthoaracic echocardiogram demonstrating the plane of the left ventricular outflow tract, the dehisced aortic valve is seen to be displaced superiorly. Note the echolucent space around the point of dehiscence.

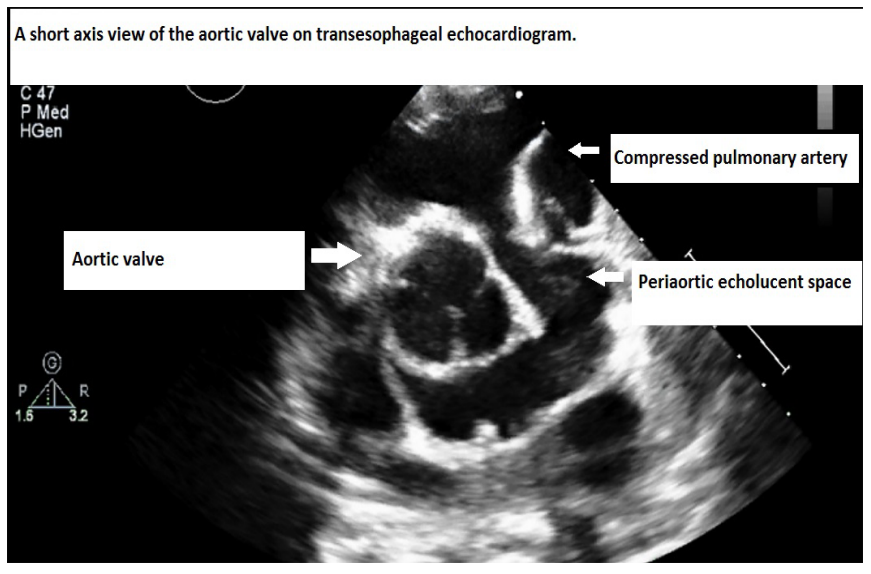

Figure 2: Short axis view of the aortic valve on transesophageal echocardiogram demonstrating a large echolucent space around the aortic valve and compression of the pulmonary trunk.

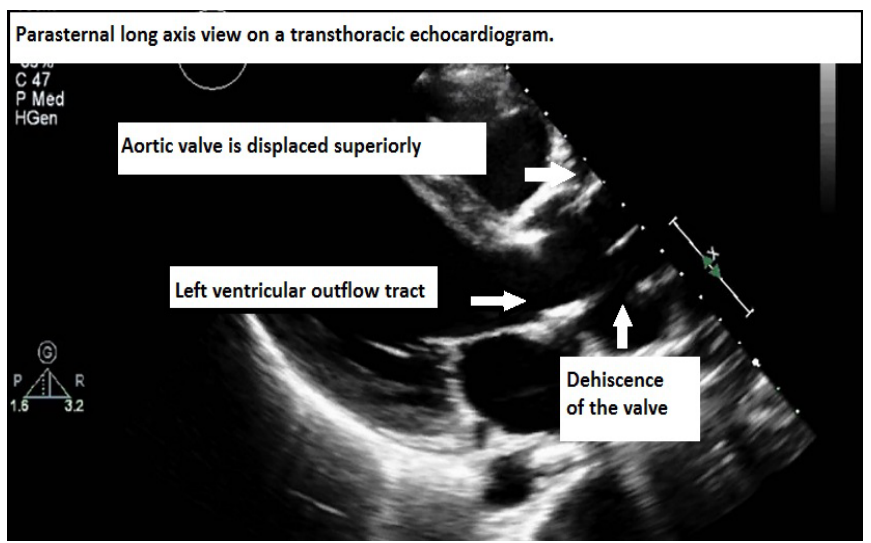

Figure 3: Color doppler mode on a short axis view of the aortic valve on transesophageal echocardiogram demonstrating blood flow between the left and right ventricular outflow tracts and into the peri-valvular space.

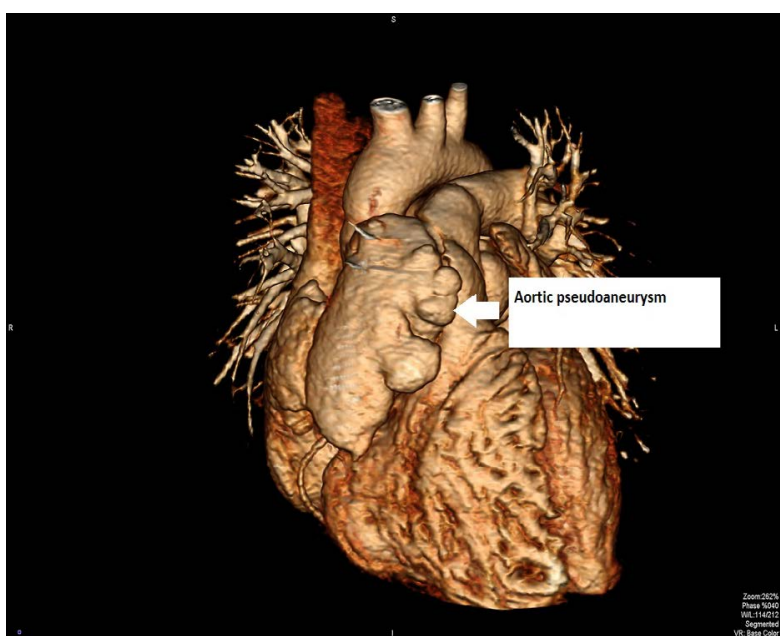

Figure 4: 3DCT demonstrating pseudoaneurysm.

\section{Discussion}

Valve dehiscence is defined as suture line breakdown leading to separation of the prosthetic valve from the annulus [1]. It is a serious complication with a high risk of mortality, historically the complication is known to occur in up to $5 \%$ of valve replacement procedures [2]. The causes of this complications can be infectious, non-infectious, degenerative or due to technical factors at the time of surgery. The most important risk factor is infective endocarditis other major risk factors include aneurysm of the ascending aorta, degenerative regurgitations and severe calcification of the native valve [2].

Less common causes include technical factors such as using continuous suture instead of interrupted sutures and noninfectious aortitis $[2,3]$.

Almost every cause of noninfectious aortitis has been reported as a cause of prosthetic valve dehiscence with a young age, high $\mathrm{C}$ - reactive protein levels and active inflammation being the most important risk factors for valve dehiscence in this setting [3]. There is one case report of aortic valve dehiscence complicating chronic granulomatous disease [4].

Patients usually present with heart failure and /or infective endocarditis, they can also present with one or more local complications of aortic valve dehiscence. The structures most likely to be involved are those in close proximity to the aortic valve such as the ascending aorta, the right ventricular outflow and mitral valve resulting in mitral regurgitation, ascending aortic dissection or right sided heart failure due to extension into the right ventricular outflow [5].

The key to diagnosis is the demonstration of a large perivalvular echolucent space with evidence of flow inside the space with or without a rocking motion of the aortic valve on imaging [6]. The imaging modalities capable of demonstrating this include transthoracic echo, transesophageal echo and computed tomographic scan [1]. A tilting of the aortic valve prosthesis by eight degrees or more on a plain chest roentgenogram is a cause for concern and requires further imaging [1]. Echo provides information about the hemodynamic consequences of the dehiscence and local effects on surrounding anatomic structures such as involvement of the mitral annulus causing mitral regurgitation [5] and compression of the pulmonary trunk as was present in this patient. On a computed tomographic scan valve dehiscence is seen as a continuous column of contrast in the perivalvular space [1]. CT allows 
Citation: Edupuganti MMR (2015) Right Sided Heart Failure-An Unusual Presentation of Aortic Valve Dehiscence. J Health Med Informat 6: 195. doi:10.4172/2157-7420.1000195

Page 3 of 3

planning for surgery by providing information about the proximity of the thoracic aneurysm to the chest wall and other surrounding structures such as coronary bypass grafts [7].

The treatment of choice is expeditious surgical replacement of the valve, repair of any associated complication such as an involvement of other valves and reimplantation of coronary arteries if possible or surgical bypass if reimplantation is not possible due to friability of tissue. The 30 day mortality for the surgery is as high as $27 \%$ and the 6 year survival rate only 13.5\% [2]. Expeditious surgery may not be possible in setting of complications from septic embolization such as mycotic aneurysms and hemorrhagic strokes [8].

\section{References}

1. Pham N, Zaitoun H, Mohammed TL, DeLaPena-Almaguer E, Martinez F, et al. (2012) Complications of aortic valve surgery: manifestations at CT and MR imaging. Radiographics 32: 1873-1892.

2. Rizzoli G, Russo R, Valente S, Mazzucco A, Valfré C, et al. (1984) Dehiscence of aortic valve prostheses: analysis of a ten-year experience. Int J Cardiol 6: 207-221.
3. Sato S, Fujita T, Oda T, Shimahara Y, Tanaka H, et al. (2014) Aortic Valve Replacement For Patients With Aortitis - Perioperative Management And Longterm Results. AATS, Aortic symposium.

4. Bhagat M, Steinberg JA, Silvestry F, Surrey L, Apter AJ, et al. (2014) Suspected non infectious prosthetic valve inflammatory dehiscence $X$ - linked chronic granulomatous disease. Journal of allergy and clinical immunology 133: 252.

5. Ivens EL, Thompson CR, Moss RR, Munt BI, Ling H (2008) Images in cardiovascular medicine: prosthetic aortic valve and conduit dehiscence with large periconduit cavity, ascending aortic aneurysm and severe mitral regurgitation. Eur J Echocardiogr 9: 148-151.

6. Armstrong WF, Ryan T (2010) Figenbaums Echocardiography. $7^{\text {th }}(E d n): 385$ 424

7. Kucher N, Kipfer B, Seiler C, Allemann Y (2004) Giant anastomotic pseudoaneurysm complicating aortic xenograft replacement. Ann Thorac Surg 77: 2197-2199.

8. Gebhard C, Biaggi P, Stähli BE, Schwarz U, Felix C (2013) Complete graft dehiscence 8 months after repair of acute type A aortic dissection. Eur Heart $J$ Acute Cardiovasc Care 2: 72-76. 